PAPER

\title{
Improved finite samples resampling for unbiased wide-band direction of arrival estimation
}

\author{
Ahmed Desoki*, Jun-ichi Takada ${ }^{\dagger}$ and Ichiro Hagiwara ${ }^{\ddagger}$ \\ Graduate School of Science and Technology, Tokyo Institute of Technology, \\ 2-12-1 Ookayama, Meguro-ku, Tokyo, 152-8552 Japan
}

(Received 13 November 2008, Accepted for publication 20 May 2009)

\begin{abstract}
This paper considers wide-band DOA estimation using the spatial resampling method for coherent averaging. In this paper a rough estimate of the resampling error distribution is obtained. Careful examination of this distribution yields an improved resampling formula suitable for, but not limited to, arrays with finite sensors. It is shown that the proposed formula yields resampling error much less than that of conventional resampling. Moreover it is shown how the resampling error can be more reduced by proper choice of the focusing frequency. Based on the this resampling formula a perfect focusing scheme is then proposed and its performance is evaluated and compared to other wide-band methods through simulations. Simulations showed quite satisfactory and robust performance of the proposed scheme. It was shown that it succeeds in situations where all considered wideband methods fail. In addition it is bias-free and can be implemented quite efficiently. Hence it is quite suitable for reliable real-time DOA estimation in reverberant environments.
\end{abstract}

Keywords: Focusing, Coherent averaging, Resampling, Finite samples, Wide-band, Direction of arrival, Virtual array, Real-time, Reverberation, Coherent

PACS number: 43.60.Gk [doi:10.1250/ast.30.417]

\section{INTRODUCTION}

For many years, sensor arrays have been used to send and receive data wirelessly. A sensor array has several advantages over a single sensor. First, it can increase the Signal to Noise Ratio (SNR). Second, sensor arrays can steer the transmitting/receiving beams and by doing that, they can send/separate multiple signals simultaneously. This is very useful in many applications such as wireless communications, acoustics and seismic applications [1-3]. For these applications Direction Of Arrival (DOA) estimation of target signals is essential for their detection and localization. In acoustics example applications of DOA estimation include automated systems for video conferencing, surveillance and hands free speech acquisition [2,5]. Industrial applications include acoustic mapping of machines and vehicles for the identification of noisy regions or parts [4]. Military applications include localization of enemy's ground vehicles, ships and submarines [6]. Localization data can also be used indirectly for other

\footnotetext{
*e-mail: desoki.a.aa@m.titech.ac.jp

†e-mail: takada@ide.titech.ac.jp

‡e-mail: hagiwara.i.aa@m.titech.ac.jp
}

tasks such as dereverberation of speech, fault prediction and analysis of machinery, cuing and tracking of cameras, speaker verification, etc. [5].

Signal subspace methods are popular DOA estimation methods designed for multiple sources and are characterized by their super-resolution. However these methods are valid only for narrow-band signal model and fail for highly correlated signals. Hence they cannot be directly applied in many situations. One example is acoustic environments which are usually wide-band and reverberant.

Wide-band generalizations of signal subspace methods can be categorized as either coherent [7] or incoherent [10]. Coherent methods perform a preprocessing step called focusing after which narrow-band techniques can be applied. In comparison to incoherent methods, advantages of coherent methods include their ability to handle highly correlated, and even coherent, signals, statistical stability and less measurement and computational time [7-9]. The first coherent averaging method was introduced by Wang and Kaveh [7] and is called Coherent Signal-Subspace Method (CSM). The main shortcoming of this method is its need for preliminary estimates of the unknown DOA's. The estimation performance of CSM is sensitive to these initial values. In fact, poor initial values can lead to biased 
estimates [11]. Many methods have been published on how to estimate focusing matrices as in $[7,12,13]$. However every one of these methods assumes a specific sources arrangement.

This led to the emergence of a class of coherent methods, called Perfect Focusing (PF) methods, that do not need preliminary DOA's estimates and hence are bias-free. The first attempt to PF was introduced by Bienvenu et al. [14] who suggested the generation of virtual arrays at every narrow-band frequency $f$ whose dimensions are proportional to $1 / f$. Output of these virtual arrays could be calculated by interpolating the physical array output. Krolik and Swingler in [15] suggested multi-rate resampling techniques, designed for digital audio and image processing, for interpolating a physical Uniform Linear Array (ULA). Later in [16] they proposed an optimum resampling minimax filter more suitable for finite sensor arrays. Other methods were introduced by Friedlander $e t a l$. [17] and Doron et al. [18] for physical array interpolation. These methods do not necessitate uniform physical arrays and can be applied on arbitrary shaped arrays provided that they satisfy the sampling theorem.

Since then, to our best knowledge, no published research applied PF by array interpolation anymore except for that of Sidorovich et al. [19] and Pham et al. [20]. Although Sidorovich [19] showed satisfactory performance of Friedlander's method, Pham in [20] obtained very poor performance of the Array Manifold Interpolation (AMI) method [18]. Moreover Chen in [24] obtained poor performance using the spatial resampling method as compared to the much better performance reported in [15] and [16].

In this paper, $\mathrm{PF}$ by spatial resampling $[15,16]$ is considered. An improved resampling formula is proposed that shows superior performance as compared to resampling methods suggested in $[15,16]$. Based on this improved formula a PF scheme is then proposed. This proposed schemes is then compared to the conventional PF methods of $[15,16]$ in addition to other wide-band DOA estimation methods. It is shown that the proposed scheme has superior and robust performance and succeeds in situations where all considered wide-band methods fail. Thus by using properly designed arrays [34], the proposed scheme can yield any desired quality of DOA estimation and poor performance due to the usage of non-suitable arrays can be avoided.

Among array interpolation methods [15-18], interpolation by spatial resampling is chosen for many reasons. Firstly, the spatial resampling method is very simple and hence can be straightforwardly used for 2D DOA estimation and 3D localization, as compared to the more complex transformations in [17] and [18] whose extension is much more difficult. Secondly, since all interpolation methods necessitate the satisfaction of the sampling theorem, uniform arrays can give the highest aperture for the same number of sensors in addition to their ease of manufacturing. Moreover the spatial resampling method can still generate any arbitrarily shaped (same dimensional) virtual arrays from the uniform physical array enabling the usage of any optimized/preferred array shape. This provides more flexibility than Friedlander's method which end with 1D ULA's generated from 2D arbitrary shaped physical arrays [25]. In other words, the necessity of uniform arrays can be considered as an advantage of, rather than a constraint on, the spatial resampling method. Thirdly, the spatial resampling method is completely applicable over the entire sensor array and for the full range of DOAs as compared to array sectors in [17] and the finite DOAs range of the AMI method [26]. Furthermore, the AMI method's focusing matrices are not unitary and hence part of the noise subspace can be confused as signal subspace [12].

Among recent wide-band DOA estimation methods that can avoid preliminary DOA estimates are the Weighted AVErage of Signal-subspaces (WAVES) [21] and the Test of Orthogonality of Projected Subspaces (TOPS) [22]. Although WAVES can avoid preliminary estimates by using a beamforming invariance technique [23], its performance is worse than when it uses focusing matrices with good initial estimates [21]. The TOPS method is however categorized as incoherent method and hence can not replace coherent ones.

This paper is organized as follows. In section 2 theoretical background of PF by spatial resampling and resampling schemes are reviewed. In section 3, a rough estimate of resampling error distribution is obtained. Careful examination of this distribution yields a new improved resampling formula suitable for arrays with finite sensors. Based on this formula, a perfect focusing scheme is then derived. In section 4 several simulations and statistical measures evaluating the proposed scheme are presented. Finally section 5 concludes this paper.

\section{THEORETICAL BACKGROUND}

\subsection{Wide-band Array Processing by Spatial Resam- pling}

For wide-band source signals, signal received by the $m$ th sensor of an $M$ identical sensor linear array can be written in the frequency domain as

$$
P_{m} \equiv P\left(x_{m}, f\right)=\sum_{k=1}^{K} U_{k}(f) e^{j \pi \tilde{x}_{m}(f) \sin \phi_{k}}+N_{m}(f),
$$

where $f$ is the temporal frequency, $U_{k}(f)$ is the $k$-th source signal in the frequency domain, $\phi_{k}$ is its angle of arrival measured from the normal to the line joining the sensors, $\tilde{x}_{m}(f) \equiv x_{m} /(\lambda / 2)=2 f x_{m} / c$ is the $m$-th sensor position normalized by half wavelength $\lambda / 2, N_{m}(f)$ is 
the frequency domain noise signal generated at the that sensor and $c$ is the propagation speed. It is clear that Eq. (1) depends on the normalized sensor position rather than the position itself. Although the physical position $x_{m}$ is constant, the normalized position $\tilde{x}_{m}(f)$ is proportional to frequency. This in turn means that covariance matrices at different frequencies can not be averaged as they are considered for different sensor arrays. This is the source of pain for which narrow-band DOA techniques cannot be directly applied to wide-band signals.

Bienvenu in [14] overcame this difficulty by using a set of virtual arrays instead of the physical array. Positions of virtual arrays' sensors, $x_{m}^{v}$, were chosen so that their normalized positions, $\tilde{x}_{m}^{v}$, remain independent on frequency. That is

$$
\tilde{x}_{m}^{v} \equiv \frac{2 f}{c} x_{m}^{v}(f)=\text { const }=\frac{2 f_{0}}{c} x_{m},
$$

where $f_{0}$ is some reference frequency called the focusing frequency and $x_{m}$ denotes the physical sensor position. From now on the superscript " $v$ " will be used to denote properties related to the virtual arrays. Thus it is clear that virtual sensors' positions are inversely proportional to frequency

$$
x_{m}^{v}(f)=\frac{f_{0}}{f} x_{m} .
$$

Thus at a frequency $f$, the wave field received by the corresponding virtual array can be written as

$$
\begin{aligned}
P_{m}^{v} & \equiv P\left(x_{m}^{v}(f), f\right)=\sum_{k=1}^{K} U_{k}(f) e^{j \pi x_{m}^{v} \sin \phi_{k}}+N_{m}^{v}(f) \\
& =\sum_{k=1}^{K} U_{k}(f) e^{2 j \pi x_{m} f_{0} \sin \phi_{k} / c}+N_{m}^{v}(f),
\end{aligned}
$$

where $N_{m}^{v}(f)$ denotes the noise signal generated at the $m$-th virtual sensor in the frequency domain. Thus for different frequencies, the corresponding covariance matrices can be coherently averaged (focused) to obtain the focused covariance matrix defined by

$$
\mathrm{R}^{\mathrm{F}}=E\left[\frac{1}{n_{f}} \sum_{f} \mathrm{P}^{v}(f)\left(\mathrm{P}^{v}(f)\right)^{\dagger}\right],
$$

where

$$
\mathrm{P}^{v}(f)=\left[P_{1}^{v}(f), P_{2}^{v}(f), \ldots, P_{M}^{v}(f)\right]^{\mathrm{T}},
$$

the superscript "F" denotes focused covariance matrix and $n_{f}$ is the number of narrow-band frequency components within the considered wide frequency band. Thus any narrow-band DOA estimation method can be applied on the focused covariance matrix (5) using the focusing frequency $f_{0}$ [14].

\subsection{Calculation of Virtual Arrays' Output by Spatial Resampling}

Output of virtual sensors, $P_{m}^{v}$, can be calculated by interpolating the output of the physical sensors, $P_{m}$, [14]. Considering Eq. (3) for DOA estimation over the wideband $f \in\left[f_{\min }, f_{\max }\right], f$ should be kept so that $f_{0} \leqslant f$ to avoid extrapolation. Usually the equality

$$
f_{0}=f_{\min }
$$

is chosen [15]. However, Chen in [24] performed his simulations using the mean frequency. This may be one of the reasons for his obtained poor performance.

For the case of a ULA with physical spacing $d$ and sensor positions $x_{m}=m d$, to avoid spatial and temporal aliasing, both the conditions $d \leqslant \lambda_{\min } / 2=c /\left(2 f_{\max }\right)$ and $f_{\text {sampling }} \geqslant 2 f_{\max }$ must be satisfied, where $\lambda_{\min }$ is the minimum wave length corresponding to $f_{\max }$ and $f_{\text {sampling }}$ is the temporal sampling frequency. Under these condition and for the special case when $d=c /\left(2 f_{\max }\right)$, Shannon's sampling theorem [27] guarantees that the continuous band-limited function $P(x) \equiv P(x, f)$ can be exactly recovered from its samples $P_{m} \equiv P\left(x_{m}, f\right)$ by the formula

$$
P(x)=\sum_{m=-\infty}^{\infty} P_{m} \operatorname{sinc}(x / d-m),
$$

where $\operatorname{sinc}(x) \equiv \sin (\pi x) / \pi x$ is the normalized sinc function. Thus output at the virtual arrays, $P_{m}^{v}$, can then be obtained by substituting $x=x_{m}^{v}$ and Eq. (3) in Eq. (8).

\subsection{Resampling of Infinite Sequence of Samples}

In some applications such as digital audio and computer graphics and imaging, original samples $P_{m}$ are usually too many so that they can be considered infinite. In practice however not all the available samples are used in evaluating Eq. (8). The following truncation is used instead [27]

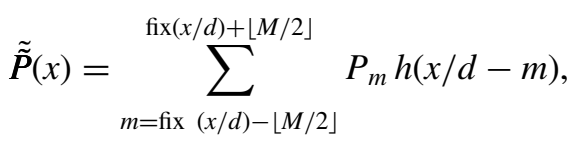

where $\tilde{\tilde{P}}(x)$ denotes the approximate value of $P(x), h$ is a finite impulse response (FIR) filter of order $M$ and linear phase, $M$ is some odd positive integer, $\operatorname{fix}(x / d)$ is the integer next to $x / d$ in the zero direction and $\lfloor M / 2\rfloor$ is the integer next to $M / 2$ in the $-\infty$ direction. Note that for the current case of infinite sequence of samples, $M$ is the filter order rather than the total number of samples. From now on the over script " $\approx$ " will be used to indicate the approximate value.

The standard problem of optimum filter design is beyond the scope of this paper. Meijering in [28] showed that the Kaiser windowed sinc filter is one of the best interpolation filters for filter order $\lfloor M / 2\rfloor \geqslant 3$. This filter is defined by 


$$
h(x)=w_{\text {Kaiser }}(x,\lfloor M / 2\rfloor d) \operatorname{sinc}(x),
$$

where $w_{\text {Kaiser }}(x,\lfloor M / 2\rfloor d)$ is the Kaiser window with half width $\lfloor M / 2\rfloor d$ defined in terms of a parameter $\alpha$. As was shown in [28] and [30], for filter orders $\lfloor M / 2\rfloor \geqslant 3$ the choice $\alpha=5$ is very satisfactory in most situations. Later in [29] Meijering compared spline interpolation [36] to sampling theorem based resampling (c.f. Eq. 9) and showed that spline interpolation offers the best accuracy in terms of computational cost.

\subsection{Resampling of Finite Sequence of Samples}

For our considered problem of resampling a physical sensor array to obtain another virtual array, sensors together with their data acquisition system are usually expensive and hence they are always finite. For a ULA symmetric with respect to the origin and with finite odd number of sensors $M$, we are obliged to truncate Eq. (8) to only use the available samples at the $M$ sensors

$$
\tilde{\tilde{P}}(x)=\sum_{m=-\lfloor M / 2\rfloor}^{\lfloor M / 2\rfloor} P_{m} h(x / d-m) .
$$

Note that the summation limits of Eq. (11) is fixed in comparison to the moving limits of Eq. (9). Thus Eq. (9) can be considered a special case of Eq. (11) when fix $(x / d)=0$, or in other words when $-1<x / d<1$.

Krolik and Swingler in [15] suggested multi-rate resampling techniques, designed for infinite sample sequences, for implementing Eq. (11). Later in [16] they proposed an optimum linear shift-variant filter more suitable for finite sensor arrays. Their filter was claimed to minimize the normalized maximum absolute resampling error at each resampled sensor at $x=x_{m}^{v}$. They showed that this optimum resampling filter is just the truncated sinc filter given by

$$
h(x)=\operatorname{sinc}(x) \equiv\left\{\begin{array}{cc}
\sin (\pi x) / \pi x, & |x| \leqslant(M-1) d \\
0, & \text { otherwise }
\end{array}\right.
$$

\section{NEW RESAMPLING FORMULA}

\subsection{Resampling Error Estimation}

Considering resampling finite sequence of samples, resampling error can be evaluated by subtracting Eq. (11) from Eq. (8) as

$$
E_{\text {Total }}(x)=E_{\text {Trunc }}(x)+E_{\text {Non-sinc }}(x),
$$

where $E_{\text {Trunc }}$, called "Truncation error," is the error due to truncation and is defined as

$$
E_{\text {Trunc }}(x)=\sum_{|m| \geqslant\lfloor M / 2\rfloor+1} P_{m} \operatorname{sinc}(x / d-m)
$$

and $E_{\text {Non-sinc }}$, called "Non-sinc error," is the error due to using resampling filter other than the sinc function and is defined as

$$
\begin{aligned}
& E_{\text {Non-sinc }}(x)= \\
& \quad \sum_{m=-\lfloor M / 2\rfloor}^{\lfloor M / 2\rfloor} P_{m}[\operatorname{sinc}(x / d-m)-h(x / d-m)] .
\end{aligned}
$$

For demonstration purpose consider the special case of broadside directions of arrival $\left(\phi_{k} \approx 0\right)$. For this case, the array output $P_{m}$ can be assumed constant, c.f. Eq. (1). Thus a rough estimate of the normalized resampling error $\bar{E}_{\text {Total }}$ can be obtained as

$$
\bar{E}_{\text {Total }}(x) \equiv \frac{E_{\text {Total }}(x)}{P_{m}}=\bar{E}_{\text {Trunc }}(x)+\bar{E}_{\text {Non-sinc }}(x),
$$

where $\bar{E}_{\text {Trunc }}$ is the normalized truncation error given by

$$
\bar{E}_{\text {Trunc }}(x) \equiv \frac{E_{\text {Trunc }}(x)}{P_{m}} \approx \sum_{|m| \geqslant\lfloor M / 2\rfloor+1} \operatorname{sinc}(x / d-m)
$$

and $\bar{E}_{\text {Non-sinc }}$ is the normalized non-sinc error given by

$$
\begin{aligned}
& \bar{E}_{\text {Non-sinc }}(x) \equiv \frac{E_{\text {Non-sinc }}(x)}{P_{m}} \approx \\
& \sum_{m=-\lfloor M / 2\rfloor}^{\lfloor M / 2\rfloor}[\operatorname{sinc}(x / d-m)-h(x / d-m)] .
\end{aligned}
$$

Fortunately it was found that the infinite series in Eq. (17) can be evaluated in the closed form expression

$$
\begin{gathered}
\bar{E}_{\text {Trunc }}(x) \approx \frac{\sin \left(\pi\left(T_{1}+1\right)\right)\left[\Psi\left(\frac{T_{1}}{2}+1\right)-\Psi\left(\frac{T_{1}+1}{2}\right)\right]}{2 \pi} \\
+\frac{\sin \left(\pi\left(T_{2}+1\right)\right)\left[\Psi\left(\frac{T_{2}}{2}+1\right)-\Psi\left(\frac{T_{2}+1}{2}\right)\right]}{2 \pi},
\end{gathered}
$$

where $T_{1}=\lfloor M / 2\rfloor-x / d, T_{2}=\lfloor M / 2\rfloor+x / d$ and $\Psi(x)$ is the digamma function [31].

Thus using equations (19) and (18), $\bar{E}_{\text {Trunc }}(x)$, $\bar{E}_{\text {Non-sinc }}(x)$ and $\bar{E}_{\text {Total }}(x)$ can be easily plotted for any filter order $\lfloor M / 2\rfloor . \bar{E}_{\text {Trunc }}(x / d)$ is plotted in Fig. 1. $\bar{E}_{\text {Non-sinc }}(x / d)$ and $\bar{E}_{\text {Total }}(x / d)$ for the Kaiser windowed sinc filter of Eq. (10) is plotted in Fig. 2. For the truncated sinc filter of Eq. (12), $\bar{E}_{\text {Non-sinc }}$ is zero and hence $\bar{E}_{\text {Total }}$ is equivalent to $\bar{E}_{\text {Trunc }}$ displayed in Fig. 1.

So as expected, Fig. 2 shows that the Kaiser windowed sinc filter, as any well designed resampling filter, drastically reduces $\bar{E}_{\text {Total }}$ in the range $-1<x / d<1$, as compared to the truncated sinc filter shown in Fig. 1. This concludes that the problem of reducing the error of resampling infinite sequence of samples can be adequately solved using well designed resampling filter of moderate order. Usually $\lfloor M / 2\rfloor=3(M=7)$ is very satisfactory filter order [33]. On the other hand, the situation is different for resampling of finite sequence of samples. As shown in Figs. 1 and 2, high oscillating resampling error is 


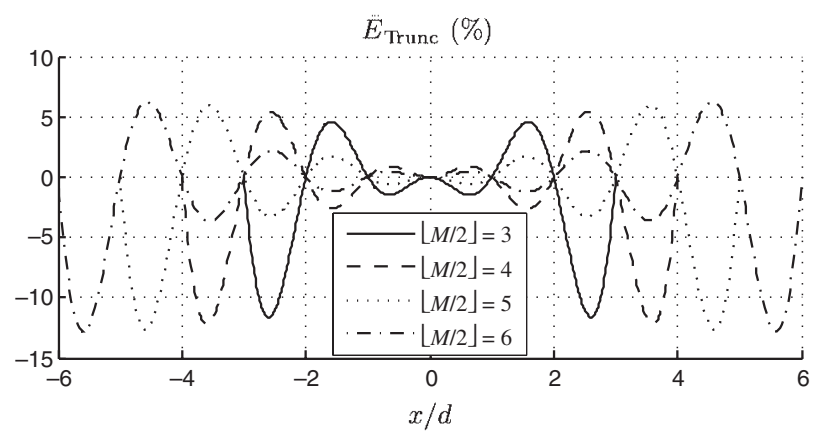

Fig. $1 \bar{E}_{\text {Trunc }}$ which is equivalent to $\bar{E}_{\text {Total }}$ for the truncated sinc filter.

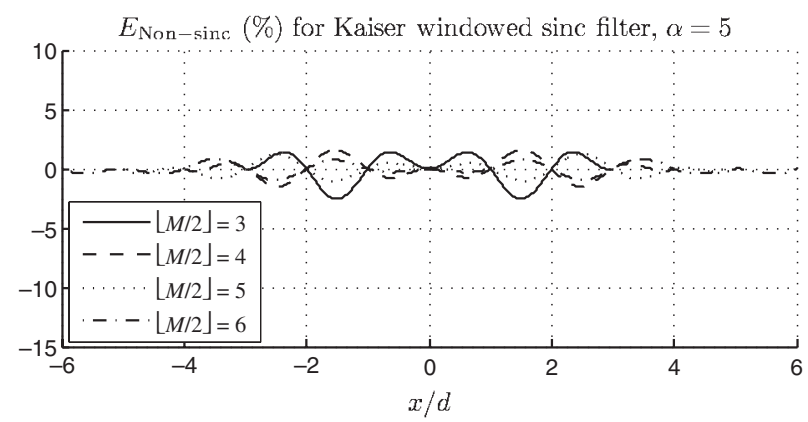

(a)

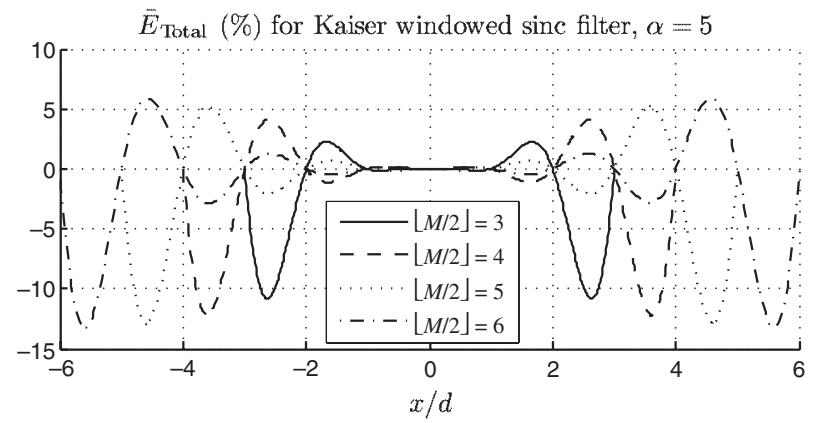

(b)

Fig. 2 (a) $\bar{E}_{\text {Non-sinc }}$ (b) $\bar{E}_{\text {Total }}$; for the Kaiser windowed sinc filter.

unavoidable near the array edges irrespective of resampling filter type or order. This is called Gibbs phenomenon [27]. One other thing to mention is that the truncated sinc filter, as shown in Fig. 1, is one of the worst filters for resampling both finite and infinite samples, as opposed to the claim in [15]. These conclusions are not our own discovery but rather well known facts valid for sampling theorem based resampling irrespective of the assumption $P_{m}=$ const $[27,33]$.

\subsection{New Resampling Formula with Drastically Re- duced Errors}

Signal subspace methods for DOA estimation are famous for their super resolution. These methods however are very sensitive to errors in estimating covariances [34]. Hence there must be a means to much more reduce resampling errors to facilitate usage of high resolution DOA estimation methods.

The use of the truncated resampling Eq. (11) instead of the exact Eq. (8) implies the assumption of zero samples outside the sensor array span, i.e.

$$
P_{m}=0 \quad \forall|m| \geqslant\lfloor M / 2\rfloor+1 .
$$

If this assumption could be realized, then Eq. (11) with the truncated sinc resampling filter would be exact. Conversely if it is invalid, resampling errors will have a distribution similar to Fig. 1.

One way to realize the assumption of Eq. (20) is to weight the finite set of samples, $P_{m} \forall|m| \leqslant\lfloor M / 2\rfloor$, with a smooth function, $w(x, b)$, which decays at both ends towards zero, to obtain the weighted sample sequence $Q_{m}$ as shown in Figs. 3(a), (b) and (c). w(x,b) denotes a weighting function with half-width $b$. As shown in Fig. 3(b), the halfwidth is set to $b=\lfloor M / 2+1\rfloor d$ not to waste edge sensors' readings. Thus as shown in Fig. 3(c), the weighted sample sequence $Q_{m}$ realizes the assumption of Eq. (20) and hence it can be exactly resampled using Eq. (11), with the truncated sinc filter, to obtain the continuous function $Q(x)$ as shown in Fig. 3(d). Finally $Q(x)$ is de-weighted using the same weighting function, Fig. 3(e), to obtain the continuous function $\tilde{\tilde{P}}(x)$ as shown in Fig. 3(f). This scheme can be cast in the form of following equation

$$
\begin{aligned}
& \tilde{\tilde{P}}(x)= \\
& \frac{\sum_{m=-\lfloor M / 2\rfloor}^{\lfloor M / 2\rfloor} P_{m} w(m d,\lfloor M / 2+1\rfloor d) h(x / d-m)}{w(x,\lfloor M / 2+1\rfloor d)} \\
& \forall|x| \leqslant\lfloor M / 2\rfloor d .
\end{aligned}
$$

The Hann window defined by

$$
w_{\text {Hann }}(x, b)=\left\{\begin{array}{lc}
0.5+0.5 \cos (\pi x / b), & |x| \leqslant b \\
0, & \text { otherwise }
\end{array}\right.
$$

is an example smooth weighting function that not only has zero ends but also has zero end slopes and smooth derivatives. Hence it will be used with Eq. (21) as it yields very accurate resampling as will be shown in the following. 3.2.1. Resampling error estimation

To validate the merit of Eq. (21), the normalized nonsinc error is calculated as was done for Eq. (18) to obtain

$$
\begin{gathered}
\bar{E}_{\text {Non-sinc }}(x) \approx \sum_{m=-\lfloor M / 2\rfloor}^{\lfloor M / 2\rfloor}[\operatorname{sinc}(x / d-m) \\
\left.-\frac{w_{\text {Hann }}(m d,\lfloor M / 2+1\rfloor d) h(x / d-m)}{w_{\text {Hann }}(x,\lfloor M / 2+1\rfloor d)}\right] .
\end{gathered}
$$

Thus the normalized total resampling error $\bar{E}_{\text {Total }}$ can be calculated using Eq. (16) with $\bar{E}_{\text {Trunc }}$ given by Eq. (19). This is shown in Fig. 4 for the truncated sinc resampling filter and Kaiser windowed sinc filter. 


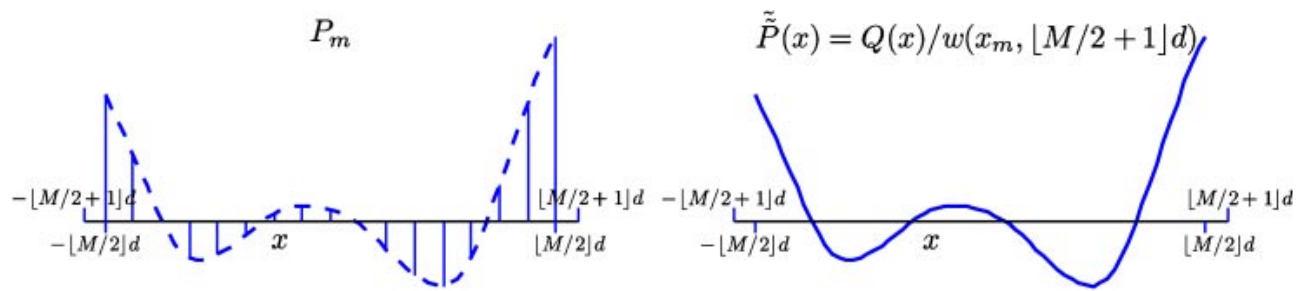

(a)

(f)

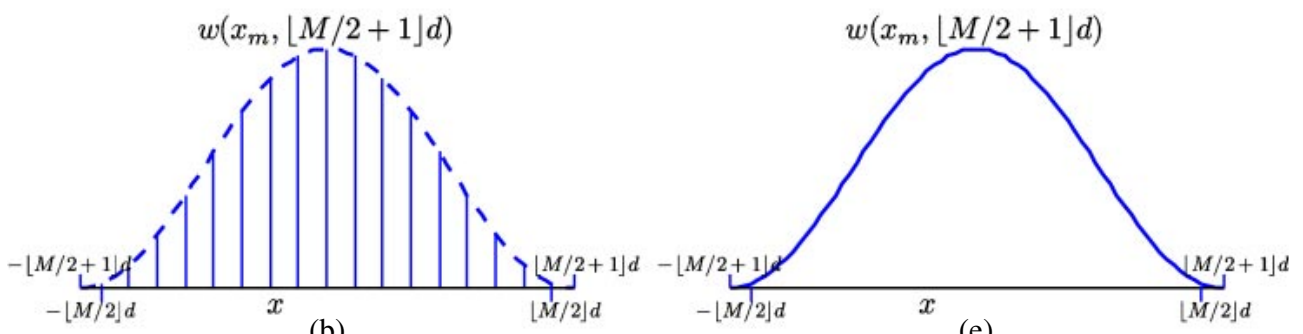

(b)

(e)

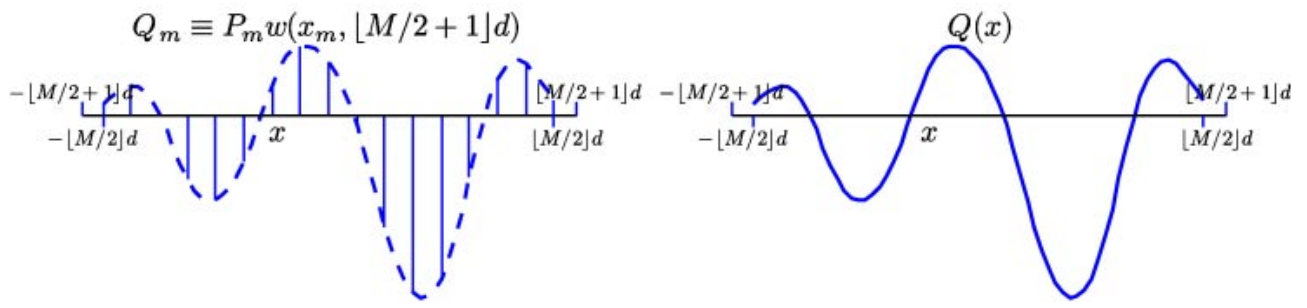

(c)

(d)

Fig. 3 New resampling scheme with drastically reduced errors; (a) Original finite samples set $P_{m}$; (b) Smooth weighting function $w\left(x_{m},\lfloor M / 2+1\rfloor d\right)$ decaying at both ends towards zero; (c) Weighted sample sequence $Q_{m} \equiv P_{m} w\left(x_{m},\lfloor M / 2+\right.$ $1\rfloor d)$; (d) The exactly resampled continuous function $Q(x)=\sum_{m=-\lfloor M / 2\rfloor}^{\lfloor M / 2\rfloor} Q_{m} \operatorname{sinc}(x / d-m)$; (e) The same weighting function is used for de-weighting $Q(x)$; (f) The resampled continuous function $\tilde{\tilde{P}}(x)=Q(x) / w\left(x_{m},\lfloor M / 2+1\rfloor d\right)$.

As shown in Fig. 4, as compared to Figs. 1 and 2, the proposed resampling formula drastically reduces resampling errors, approximately 20 times. Also it is found that the new resampling formula using the truncated sinc filter yields less error than that obtained using the known to be good Kaiser windowed sinc filter. This expected result is because Eq. (11) will be equivalent to the exact Eq. (8) using the truncated sinc filter in case the assumption (20) is valid.

\subsection{PFISR: Perfect Focusing using Improved Spatial Resampling}

Using the proposed resampling formula, Eq. (21), instead of Eq. (11) for focusing by spatial resampling will be denoted "Perfect Focusing using Improved Spatial Resampling" or shortly PFISR. From now on Eq. (21) will be always used with the truncated sinc filter as was explained in section 3.2.1.

\subsubsection{Further resampling error reduction}

More careful examination of Fig. 4 shows, as expected, that resampling error is highest near the edges. If, for example, resampling could be avoided within edge sensors, resampling error will be much more reduced. This exceptional performance can however be achieved without wasting these, precious, edge sensors.

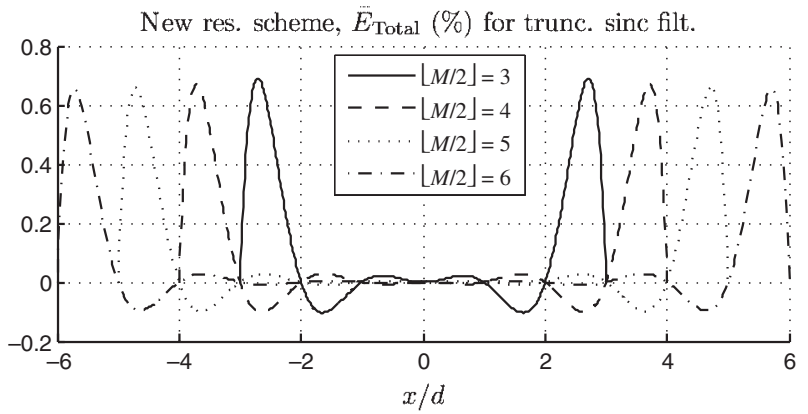

(a)

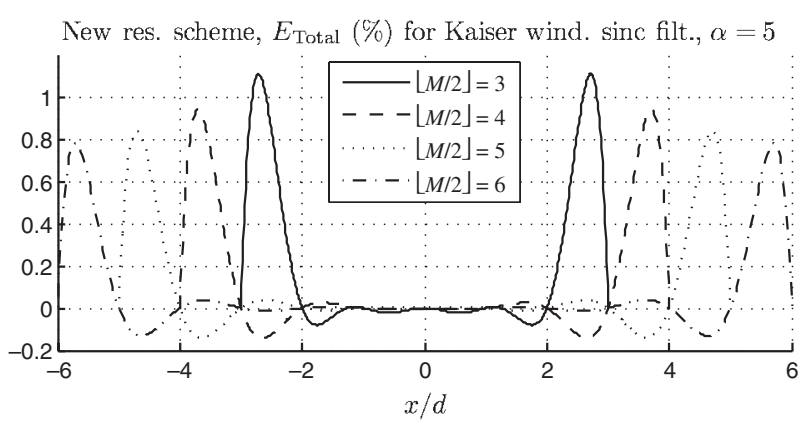

(b)

Fig. $4 \bar{E}_{\text {Total }}$ for the proposed new resampling formula using (a) the truncated sinc filter (b) Kaiser windowed sinc filter. 


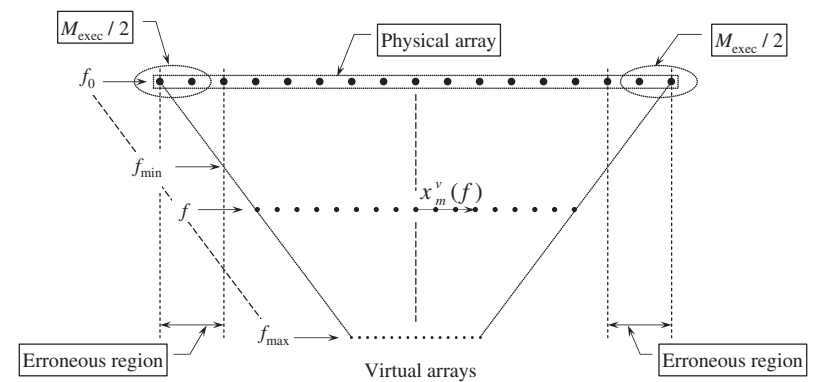

Fig. 5 Virtual arrays avoiding erroneous edge regions.

Figure 5 shows the virtual arrays distribution, Eq. (3), versus frequency. As shown virtual arrays span decreases with increasing the frequency. Let's define $M_{\text {exec }}$ as the number of sensors to be excluded from both array edges due to their large resampling error. Let's also define the "erroneous regions" to be the regions occupied by $M_{\text {exec }}$ at both edges as indicated in Fig. 5. If we constrain ourselves to use resampled arrays whose span do not intersect with these erroneous regions, their large resampling errors can then be avoided and a very low resampling error can be expected. As shown in Fig. 5 this goal can be achieved by reducing the focusing frequency $f_{0}$ below $f_{\min }$ such that

$$
f_{0}\left(M_{\mathrm{exec}}\right)=\frac{M-1-M_{\mathrm{exec}}}{M-1} f_{\min },
$$

as opposed to the usual choice of Eq. (7). From now on the focusing frequency choices of equations (7) and Eq. (24) will be denoted by $f_{0}$ and $f_{0}\left(M_{\text {exec }}\right)$ respectively.

In the following sections merit and quality of PFISR scheme will be verified. In these sections we use $M_{\text {exec }}=4$.

\section{SIMULATIONS}

Performance of the PFISR scheme is compared to other wide-band DOA estimation methods through the following examples.

\subsection{Performance of the Proposed PFISR Method}

Example 1: Consider the problem of DOA estimation of 5 white Gaussian noise sources whose angles are $\phi_{1}=0^{\circ}, \phi_{2}=8^{\circ}, \phi_{3}=13^{\circ}, \phi_{4}=33^{\circ}$ and $\phi_{5}=37^{\circ}$. The last four source angles are the same as those used in Example 1 in [12]. The reason why the first source is added to the current example will be clarified later in Example 2. The third, fourth and fifth signals are exactly delayed versions of the second one. Delays are given by $T / 8,3 T / 8$ and $5 T / 8$ where $T$ is measurement snapshot duration. The first source is however uncorrelated with them. All the five sources occupy the wide-band from $f_{\min }=6.6 \mathrm{kHz}$ to $f_{\max }=10 \mathrm{kHz}$, or from $0.66 \pi$ to $\pi$ in the $\omega$ domain which is analogous to Example 1 in [12]. A 15 sensor ULA whose sensor spacing is half wave length of the maximum frequency is used.

Fast Fourier Transform (FFT) is performed with maximum frequency of $f_{\max }$ and $n_{\mathrm{FFT}}=201$ narrow-band frequency component per FFT (measurement) snapshot. Averaging is performed using 20 measurement snapshots. Signal to Noise Ratio (SNR) is set to zero at a single frequency component at $f=f_{\min }$. i.e., pure noise exists at this frequency component. This is set intensionally to make the simulations more realistic.

Four other methods are tested for comparison with the proposed PFISR scheme: spatial resampling using truncated sinc resampling filter [16], spatial resampling using Kaiser windowed sinc resampling filter [15], spatial resampling using cubic spline interpolation and CSM [7]. The PFISR method is simulated twice using $f_{0}=$ $f_{\min }=6,600 \mathrm{~Hz}$ and $f_{0}\left(M_{\mathrm{exec}}=4\right)=4,700 \mathrm{~Hz}$ in accordance with Eq. (7) and (24) respectively. For the CSM method $f_{0}$ was set to $8300 \mathrm{~Hz}$ which is the mean frequency $[7,12]$. For the residual methods the focusing frequency was set to $f_{0}=f_{\min }=6600 \mathrm{~Hz}$. About CSM focusing, the strategy proposed in [12] was used as it was claimed to yield substantially better performance than that in [7] for multi-group sources as in the current example. That is, 1) Coarse Resolution: Obtain initial DOA estimates $\beta_{i}$ 2) Fine Resolution: Use $\beta_{i}$ and $\beta_{i} \pm 0.25 \mathrm{BW}$ as focusing angles, where $\mathrm{BW} \equiv 2 /(M-1)$ is the beamwidth of the array at the frequency whose corresponding half wavelength is the sensor spacing. About the initial DOA estimates $\beta_{i}$, they were chosen at $0^{\circ}, 10.5^{\circ}$ and $35^{\circ}$, which are the exact first DOA and the means of the following successive DOA pairs. These estimates are the best expected using delay and sum beamforming. Higher resolution methods such as Capon's [3] were not used for $\beta_{i}$ estimation since they fail for correlated sources. Using $\mathrm{SNR}=10 \mathrm{~dB}$, resampling error and DOA estimation results using the MUltiple SIgnal Classification (MUSIC) method are calculated and plotted in Figs. 6, 7, 8 and 9. In Figs. 8 and 9, DOA estimation using the virtual arrays' exact output, Eq. (4), was also calculated and plotted for $f_{0}=4,700,6,600$ and $8,300 \mathrm{~Hz}$ as benchmarks for their respective coherent averaging methods.

As shown in Figs. 6(c) and (d) and Fig. 7(a), spline interpolation outperforms sampling theorem based resampling as was reported in [29]. Also Figs. 6(a) and (b) show how the proposed resampling formula, Eq. (21), used with $f_{0}\left(M_{\text {exec }}\right)=4$, Eq. (24), yields resampling error less than that obtained by using $f_{0}$, Eq. (7). For both choices of $f_{0}$, it is clear how the resampling error of the proposed formula is much less than those of the truncated sinc, Kaiser windowed sinc resampling filters and even cubic spline interpolation. Note how resampling error of the proposed formula is drastically reduced at edges. This is why the 


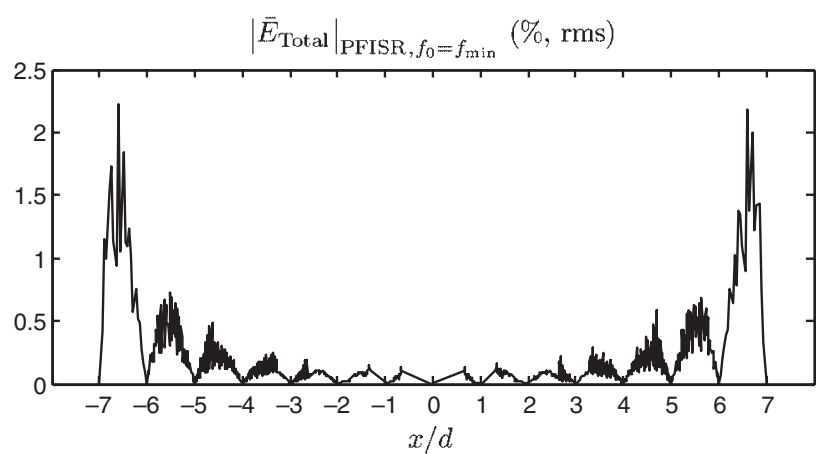

(a)

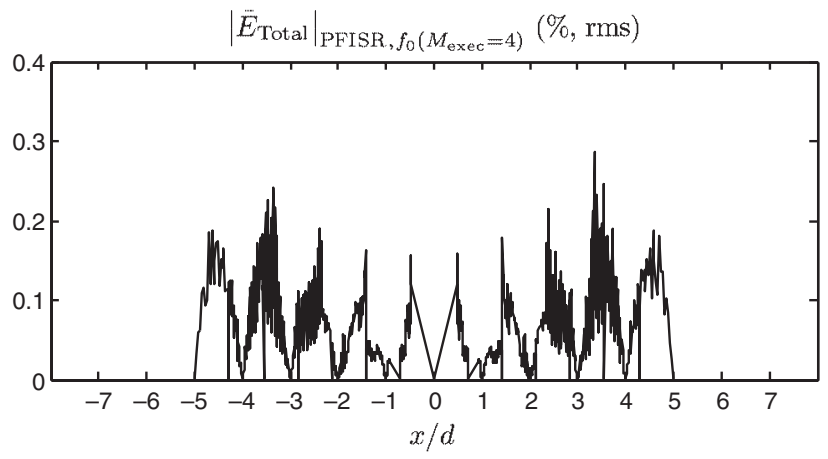

(b)

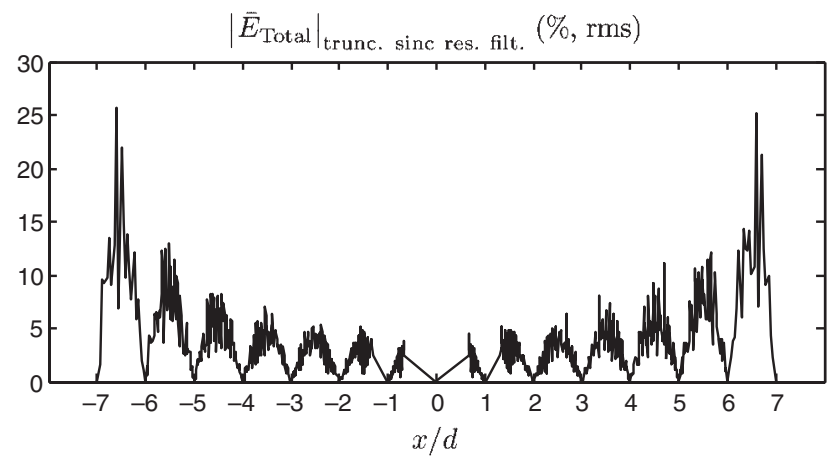

(c)

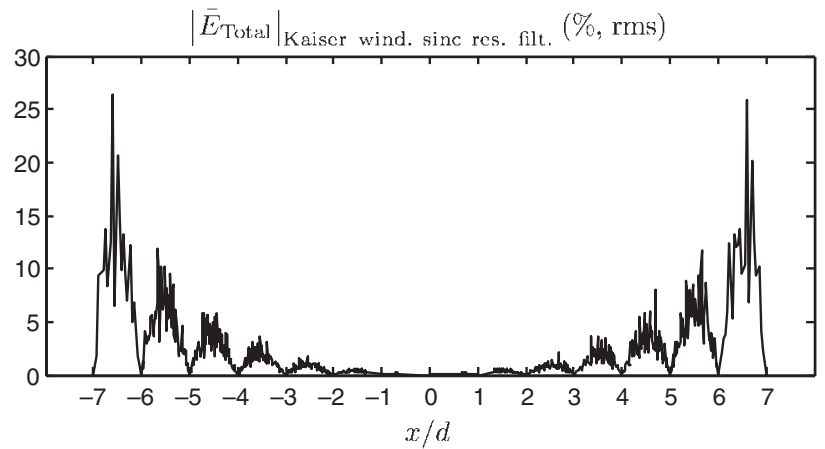

(d)

Fig. 6 Actual percentage $\left|\bar{E}_{\text {Total }}\right|$ (rms) for resampled virtual arrays using PFISR at both (a) $f_{0}$ and (b) $f_{0}\left(M_{\text {exec }}=4\right)$, (c) Truncated sinc resampling filter and (d) Kaiser windowed sinc resampling filter, where $\lfloor M / 2\rfloor=7$.

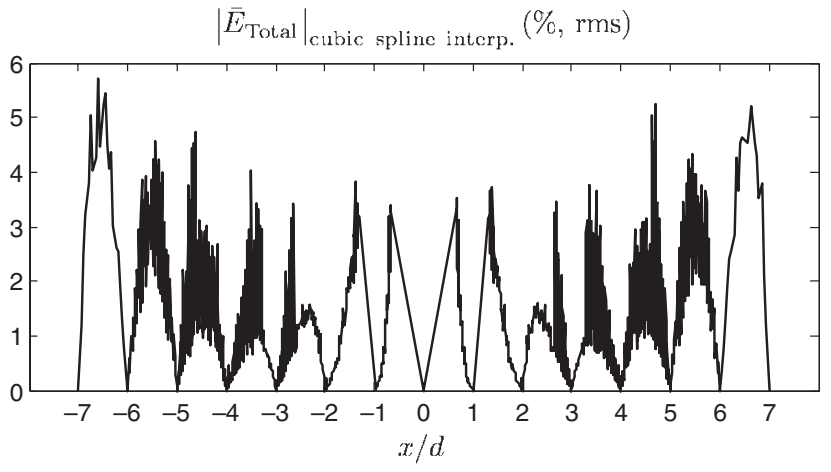

(a)

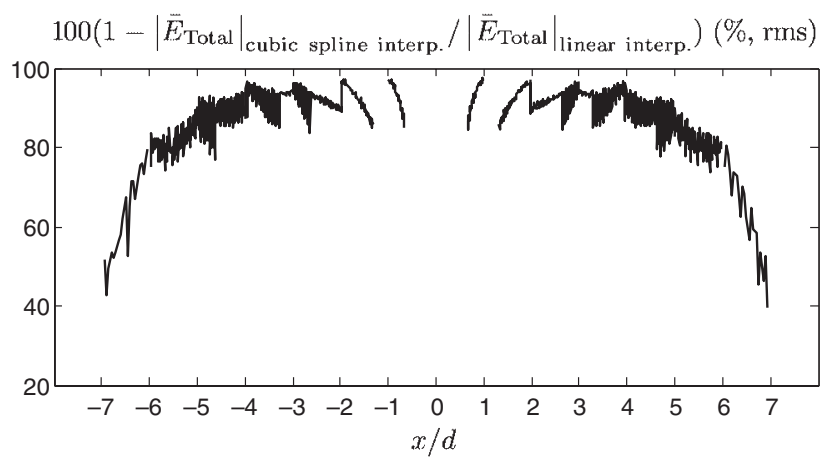

(b)

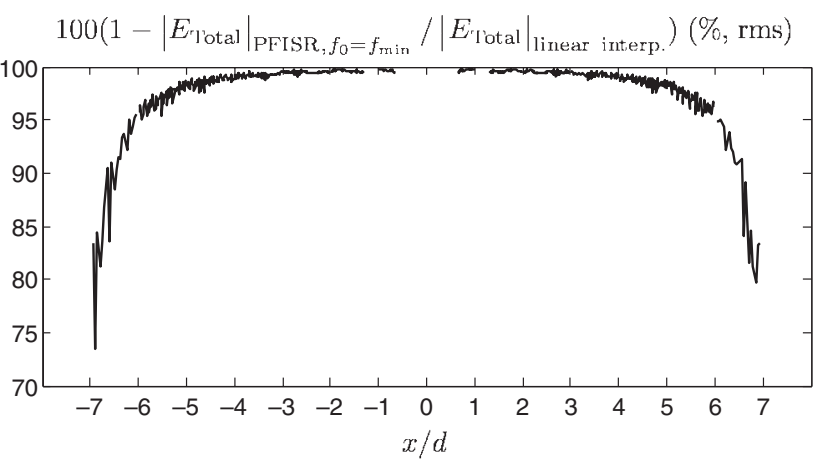

(c)

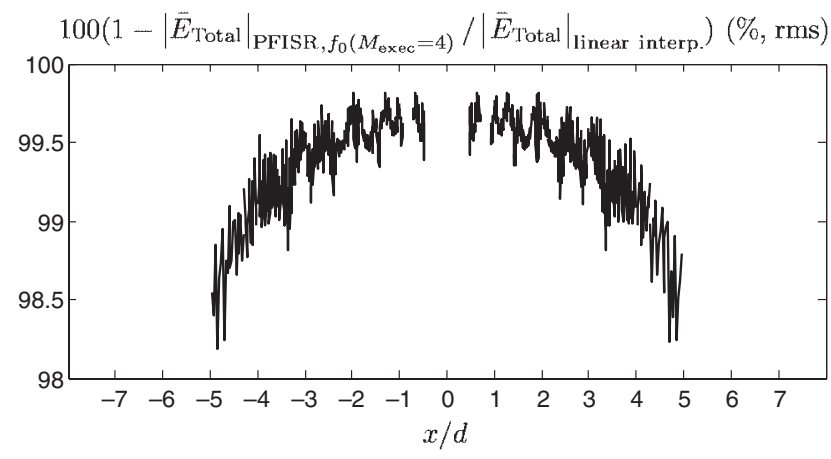

(d)

Fig. 7 (a) Actual percentage $\left|\bar{E}_{\text {Total }}\right|$ (rms) for resampled virtual arrays using cubic spline interpolation, Percentage resampling error reduction (rms) for (b) Cubic spline interpolation, (c) PFISR at $f_{0}$ and (d) PFISR at $f_{0}\left(M_{\text {exec }}=4\right)$, as compared to linear interpolation. 


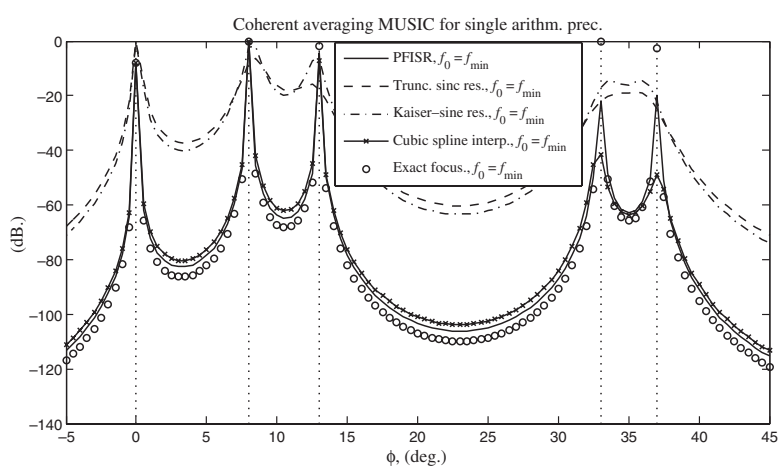

Fig. 8 PFISR at $f_{0}=f_{\min }$ as compared to truncated sinc and Kaiser windowed sinc resampling filters and cubic spline interpolation.

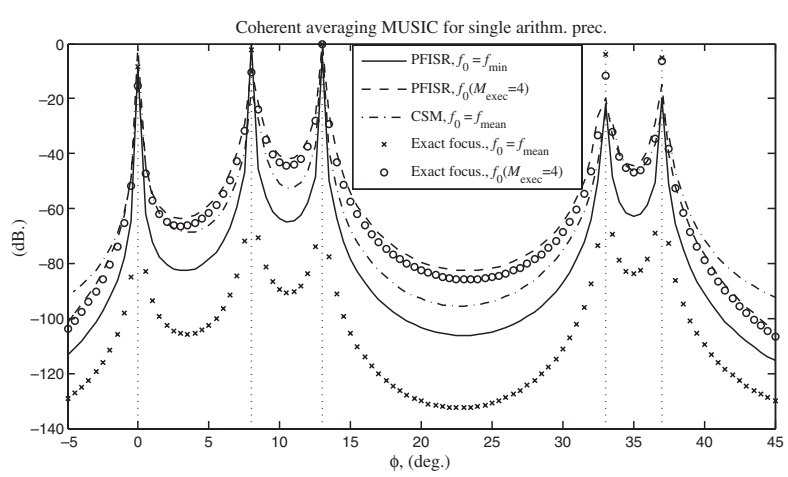

Fig. 9 PFISR at $f_{0}=f_{\min }$ and $f_{0}\left(M_{\text {exec }}=4\right)$ as compared to CSM.

PFISR performance is very close to its ideal benchmark and is better than that of cubic spline interpolation and much better than that of truncated sinc and Kaiser windowed sinc resampling filters, as shown in Figs. 8 and 9. Also note how the (claimed to be optimum [16]) truncated sinc resampling filter yields the worst resampling error and weakest DOA estimation. On the other hand Fig. 9 shows good performance of the CSM method as was reported in [12]. However this is not always the case, as will be seen in example (2).

As a further analysis of the proposed resampling formula, Figs. 7(b), (c) and (d) show error reduction of cubic spline interpolation and the proposed resampling formula as compared to linear interpolation. These figures can be compared with the error estimates given in [29] in the range $-1<x / d<1$, as was explained in section 2.4. Within this range cubic spline interpolation yielded in Fig. 7(b) a minimum error reduction around $84 \%$, which is within the $28 \%-91 \%$ estimate reported in [29]. In contrast, within the same range the proposed resampling formula yielded in Figs. 7(c) and (d) a minimum error reduction around $99.4 \%$ for both $f_{0}$ and $f_{0}\left(M_{\text {exec }}\right)=4$. This is surprisingly higher than the $66 \%-98 \%$ error reduction estimate obtained in [29] for higher-degree spline interpolation, where splines as high-degree as nonic (9th

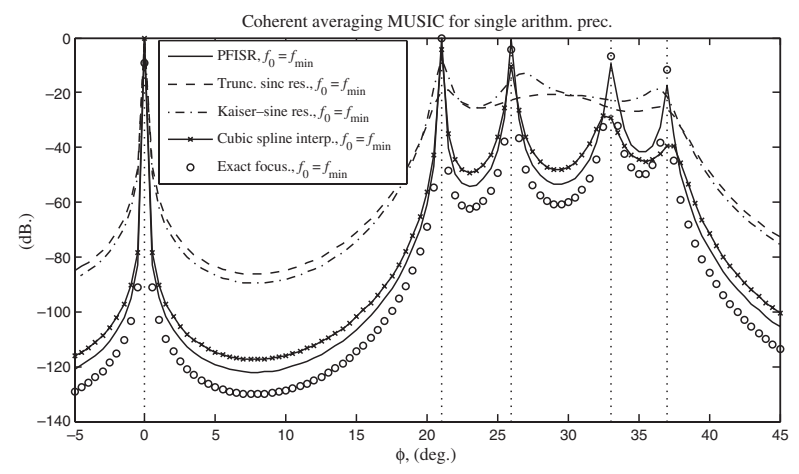

Fig. 10 PFISR at $f_{0}=f_{\min }$ as compared to truncated sinc and Kaiser windowed sinc resampling filters and cubic spline interpolation.

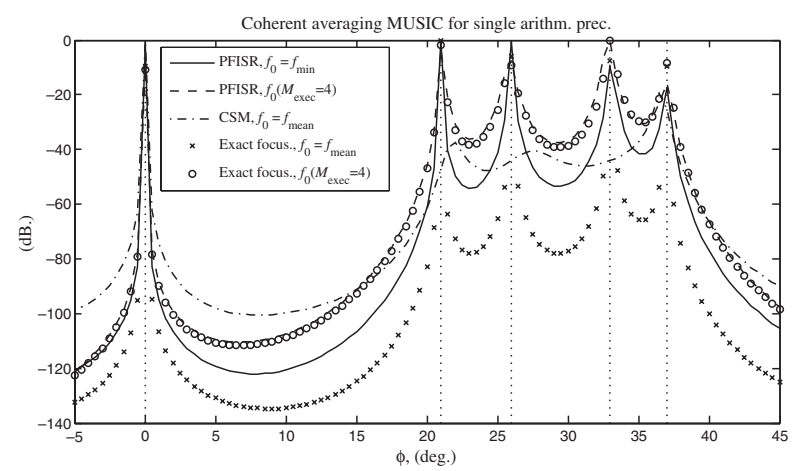

Fig. 11 PFISR at $f_{0}=f_{\min }$ and $f_{0}\left(M_{\text {exec }}=4\right)$ as compared to CSM.

degree) were examined, albeit the increased computational cost. Note that the advantage of spline over polynomial interpolation stems from its usage of increased number of low degree polynomial pieces rather than increasing the polynomial order [36]. Thus spline interpolation avoids the problem of Runge's phenomenon which occurs when using high degree polynomials [37]. In fact the cardinal spline (spline analog of the sinc function) converges strongly to the sinc function with increasing the spline degree $[38,39]$. In this case spline interpolation will yield same artifacts obtained by sampling theorem based resampling [38].

Example 2: In this example, the second and third sources of Example 1 are only shifted $13^{\circ}$ to become at $\phi_{2}=21^{\circ}$ and $\phi_{3}=26^{\circ}$. All the conditions of Example 1 are used herein except for two additional senors are added to the sensor array to have a total of $M=17$ sensor. Hence the focusing frequency $f_{0}\left(M_{\mathrm{exec}}=4\right)$ will be changed to $4,950 \mathrm{~Hz}$ in accordance with Eq. (24). Regarding initial DOA estimates $\beta_{i}$ necessary for CSM, they were chosen at $0^{\circ}, 23.5^{\circ}$ and $35^{\circ}$ in a manner similar to Example 1. Results obtained using the MUSIC method are similarly displayed in Figs. 10 and 11. Regarding the actual resampling errors, they were found very similar to Fig. 6 and hence are not plotted herein again. 
As shown in Figs. 10 and 11, all the considered methods fail to resolve the last four sources except for the proposed PFISR method and spatial resampling using cubic spline interpolation. As shown in the figures, the PFISR method could correctly estimate all the sources DOAs with good dynamic range for $f_{0}=f_{\min }$ and reasonable one for $f_{0}\left(M_{\text {exec }}=4\right)$. Spline interpolation on the other hand yielded poor dynamic range for the last two sources and wrongly estimated the fourth source DOA. Regarding the CSM method, note how its performance deteriorates in contrast to Example 1 . The $\left(\beta_{i}, \beta_{i}-0.25 \mathrm{BW}, \beta_{i}+\right.$ $0.25 \mathrm{BW})$ initial DOAs estimates used yields the angles $\left(-1.8^{\circ}, 0^{\circ}, 1.8^{\circ}, 21.7^{\circ}, 23.5^{\circ}, 25.3^{\circ}, 33.2^{\circ}, 35^{\circ}, 36.8^{\circ}\right)$. Although they include the angles $21.7^{\circ}, 25.3^{\circ}, 33.2^{\circ}$ and $36.8^{\circ}$ ) which are very close to the the last four sources of the current example, but CSM fails to resolve them. It was found however that CSM succeeds when excluding the initial DOA estimates $\pm 1.8^{\circ}$ adjacent to the first actual DOA although they are far enough from the last four sources. This reveals how the CSM results can be seriously affected (biased) by the initial DOA estimates and this is why the first DOA, $\phi_{1}=0^{\circ}$, was added to examples 1 and 2 of this paper as compared to Example 1 in [12]. One last note is about the computational time and resources of the CSM method. The RSS [12] focusing strategy used herein requires Singular Value Decomposition (SVD) and a number of matrix multiplications for every narrow-band frequency component used to compute $\boldsymbol{R}^{\mathrm{F}}$ of Eq. (5). This is in addition to the computational burden of performing low resolution DOA estimation to obtain the initial estimates $\beta_{i}$ and then iteratively refining them as explained in $[7,12]$. In contrast the proposed PFISR method is biasfree, more robust and can succeed in situations where most wide-band methods fail, as was shown in examples (1) and (2). In addition PFISR only requires the evaluation of the light Eq. (21) for every narrow-band frequency component, which can be implemented quite efficiently using the Digital Signal Processing (DSP) hardware available during data acquisition of sensor measurements [35]. This reveals how PFISR is quite suitable for reliable real-time DOA estimation.

Finally it is clear from Figs. 6, 8 and 10 that although using $f_{0}$ of Eq. (24) yields resampling error less than that obtained by using Eq. (7), its corresponding DOA estimation performance is worse. This can be explained by noting that the normalized sensor spacing of the focusing array equals $\tilde{d}=2 f_{0} d / c$ (cf. Eq. (2)). Thus using Eq. (24) instead of Eq. (7) reduces the focusing normalized sensor spacing and hence the focusing array aperture with the ratio $\left(M-1-M_{\text {exec }}\right) /(M-1)$. This explains why focusing using Eq. (24) for $f_{0}$ is not as sharp as that using Eq. (7); refer to [34] for a detailed analysis of DOA estimation performance versus $\tilde{d}$. This is true for PFISR for which

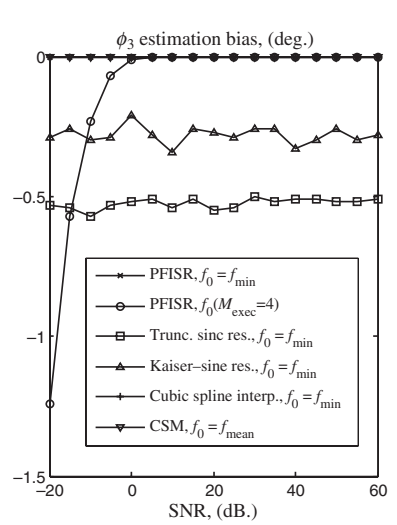

(a)

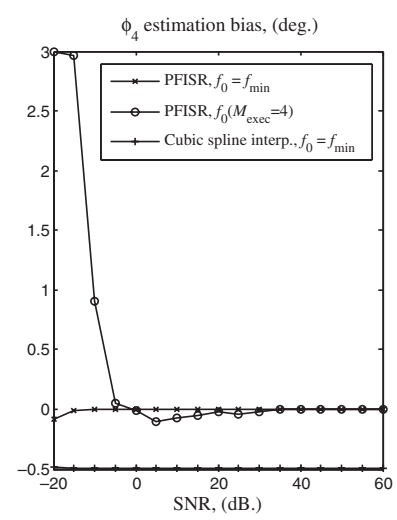

(c)

Fig. 12 Estimation bias of PFISR as compared to other methods; (a) $\phi_{3}\left(=13^{\circ}\right)$ of example (1) (b) $\phi_{2}\left(=21^{\circ}\right)$ of example (2) (c) $\phi_{4}\left(=33^{\circ}\right)$ of example (2).

the array output is approximated by Eq. (21), and even for the spatial resampling benchmarks which use the exact resampled array output, Eq. (4).

\subsection{Statistical Performance of the Proposed PFISR Method}

In this section statistical performance of the proposed PFISR method is evaluated by performing 50 Monte Carlo runs of examples (1) and (2) for different SNR's. Estimation biases for the third source of example (1) and the second and fourth sources of example (2) are shown in Fig. 12. Only PFISR and cubic spline statistics are displayed in Fig. 12(c) since they are the only methods that could detect the fourth source of example (2).

Figure 12 shows the superior stability and robustness of the proposed PFISR method, using Eq. (7) for $f_{0}$, for different situations as compared to all the other methods considered. It is shown how the extra resampling accuracy gained by using Eq. (24) is smeared in low SNR. For such a case the reduced array aperture due to using Eq. (24) can deteriorate the results as shown in the figure. It is shown also how the truncated sinc resampling filter [16] yields the worst estimation performance as compared to other 
resampling filters. It is shown also how spline interpolation overcomes sampling theorem based resampling. Finally Fig. 12(b) shows how CSM can be biased irrespective of SNR.

\section{CONCLUSION}

In this paper, an improved resampling formula suitable for, but not limited to, arrays with finite sensors was developed. This improved resampling formula yielded the PFISR DOA estimation scheme. Merit and quality of this scheme were verified by simulations applied for wide-band DOA estimation using the MUSIC method. Simulations showed quite satisfactory and robust performance of the proposed scheme. In fact it was shown that it succeeds in situations where all other considered wide-band methods fail. In addition the proposed PFISR scheme is bias-free and can be implemented quite efficiently. Hence it is quite suitable for reliable real-time DOA estimation in reverberant environments. Although it was shown how to further improve resampling accuracy by reducing the focusing frequency, this was found not good practice as it reduces the focusing array aperture and hence deteriorates the final DOA estimation performance.

This paper considers 1D ULAs for 1D DOA (bearing) estimation, it is however completely extendible for $2 \mathrm{D}$ arrays for 2D (zenith and azimuth) DOA estimation and for 3D arrays for 3D (radius, zenith and azimuth) localization of signal sources assuming spherical waves model. Finally although this research is from the perspective of acousticians, it is completely applicable on radio and seismic waves. So it is quite usable in communications and seismic applications as well.

\section{ACKNOWLEDGMENT}

The authors would like to express their deep gratitude for the referees for their valuable suggestions that much improved this paper.

\section{REFERENCES}

[1] D. Johnson and D. Dudgeon, Array Signal Processing: Concepts and Techniques (Prentice Hall, Englewood Cliffs, N.J., 1993).

[2] M. S. Brandstein and D. B. Ward, Eds., Microphone Arrays: Signal Processing Techniques and Applications (SpringerVerlag, Berlin, 2001).

[3] V. S. U. Pillai, Array Signal Processing (Springer Verlag, New York, 1989).

[4] J. J. Christensen and J. Hald, "Beamforming," Brüel \& Kjorr Tech. Rev., (1), pp. 1-48, available online @ @ttp://www. bksv.com/Library/Technical\%20Reviews.aspx (2004).

[5] Y. Huang, J. Benesty and J. Chen, "Identification of acoustic MIMO systems: Challenges and opportunities," Signal Process., 86, 1278-1295 (2006).

[6] T. Pham and B. Sadler, "Wideband acoustic array processing to detect and track ground vehicles," 1st Annu. ARL Sensors and Electron Devices Symp., pp. 151-154, College Park, MD
(1997).

[7] H. Wang and M. Kaveh, "Coherent signal-subspace processing for the detection and estimation of angles of arrival of multiple wide-band sources," IEEE Trans. Acoust. Speech Signal Process., 33, 823-831 (1985).

[8] H. Wang, C. C. Li and J. X. Zhu, "High-resolution direction finding in the presence of multipath: A frequency-domain smoothing approach," Proc. IEEE Int. Conf. Acoust. Speech Signal Process., ICASSP-87, pp. 2276-2279 (1987).

[9] J. X. Zhu and H. Wang, "A comparison of smoothing approaches for angle measurement," IEEE Trans. Aerosp. Electro. Syst., 25, 529-535 (1989).

[10] G. Su and M. Morf, "Signal subspace approach for multiple wide-band emitter location," IEEE Trans. Acoust. Speech Signal Process., 31, 1502-1522 (1983).

[11] D. Swingler and J. Krolik, "Source location bias in the coherently focused high-resolution broadband beamformer," IEEE Trans. Acoust. Speech Signal Process., 37, 143-145 (1989).

[12] H. Hung and M. Kaveh, "Focusing matrices for coherent signal-subspace processing," IEEE Trans. Acoust. Speech Signal Process., 36, 1272-1282 (1988).

[13] M. Doron and A. Weiss, "On focusing matrices for wideband array processing," IEEE Trans. Signal Process., 40, 12951302 (1992).

[14] G. Bienvenu, P. Fuerxer, G. Vezzosi, L. Kopp and F. Florin, "Coherent wide band high resolution processing for linear array," Proc. IEEE Int. Conf. Acoust. Speech Signal Process., ICASSP-89, pp. 2799-2802 (1989).

[15] J. Krolik and D. Swingler, "Focused wide-band array processing by spatial resampling," IEEE Trans. Acoust. Speech Signal Process., 38, 356-360 (1990).

[16] J. Krolik and D. Swingler, "The performance of minimax spatial resampling filters for focusing wide-band arrays," IEEE Trans. Acoust. Speech Signal Process., 39, 1899-1903 (1991).

[17] B. Friedlander, "The root-music algorithm for direction finding with interpolated arrays," Signal Process., 30, 15-29 (1993).

[18] M. Doron, E. Doron and H. Weiss, "Coherent wide-band processing for arbitrary array geometry," IEEE Trans. Signal Process., 41, 414-417 (1993).

[19] D. V. Sidorovich and A. B. Gershman, "Two-dimensional wideband interpolated root-music applied to measured seismic data," IEEE Trans. Signal Process., 46, 2263-2267 (1998).

[20] T. Pham and B. M. Sadler, "Wideband array processing algorithms for acoustic tracking of ground vehicles," Tech. Rep. US Army Res. Lab., http://www.arl.army.mil/sedd/ acoustics/reports.htm (1998).

[21] E. D. Claudio and R. Parisi, "Waves: Weighted average of signal subspaces for robust wideband direction finding," IEEE Trans. Signal Process., 49, 2179-2190 (2001).

[22] Y.-S. Yoon, L. M. Kaplan and J. H. McClellan, "TOPS: New DOA estimator for wideband signals," IEEE Trans. Signal Process., 54, 1977-1989 (2006).

[23] T. S. Lee, "Efficient wideband source localization using beamforming invariance technique," IEEE Trans. Signal Process., 42, 1376-1387 (1994).

[24] Y. Chen and F. Yu, "A comparison of spatial interpolation and transformation matrices in css for bearing estimation of multiple broadband signals," J. Acoust. Soc. Am., 90, 31493154 (1991).

[25] B. Friedlander and A. J. Weiss, "Direction finding for wideband signals using an interpolated array," IEEE Trans. Signal Process., 41, 1618-1634 (1993).

[26] M. Doron and E. Doron, "Wavefield modeling and array processing, part II-Algorithms," IEEE Trans. Signal Proc- 
ess., 42, 2560-2570 (1994).

[27] A. V. Oppenheim and R. W. Schafer, Digital Signal Processing (Prentice-Hall Inc., Englewood Cliffs N.J., 1975).

[28] E. H. W. Meijering, W. J. Niessen, J. P. W. Pluim and M. A. Viergever, "Quantitative comparison of sinc-approximating kernels for medical image interpolation," Proc. 2nd Int. Conf. Medical Image Computing and Computer-Assisted Intervention-MICCAI '99, 210-217 (1999).

[29] E. H. W. Meijering, W. J. Niessen and M. A. Viergever, "Quantitative evaluation of convolution-based methods for medical image interpolation," Med. Image Anal., 5, 111-126 (2001).

[30] T. Theussl, H. Hauser and E. Groller, "Mastering windows: Improving reconstruction," Proc. IEEE/ACM SIGGRAPH Volume Visualization and Graphics Symp., pp. 101-108 (2000).

[31] E. W. Weisstein, "Digamma function," http://mathworld. wolfram.com/DigammaFunction.html.

[32] Brüel \& Kjær, Multichannel Analysis System Type 3550, Vol. 1: Guided Tours (Brüel \& Kjær Technical Documentation).

[33] R. Sudhakar, R. C. Agarwal and S. C. Dutta Roy, "Time Domain Interpolation Using Differentiators," IEEE Trans. Acoust. Speech Signal Process., 30, 992-997 (1982).

[34] A. Desoki, J. Takada and I. Hagiwara, "Efficient array design algorithm for wide-band application of the MUltiple SIgnal Classification algorithm," Acoust. Sci. \& Tech., 30, 187-198 (2009).

[35] R. E. Crochiere and L. R. Rabiner, Multirate Digital Signal Processing (Prentice-Hall Inc., Englewood Cliffs, N.J., 1983).

[36] I. J. Schoenbcrg, "Contribution to the problem of approximation of equidistant data by analytic functions," Q. Appl. Math., 4, 45-99, 112-141 (1946).

[37] Wikipedia contributors, "Runge's phenomenon," Wikipedia, The Free Encyclopedia, http://en.wikipedia.org/w/index. php?title $=$ Runge\%27s_phenomenon\&oldid $=282944107 \quad$ (accessed Apr. 10, 2009).

[38] M. Unser, "Splines A perfect fit for signal and image processing," IEEE Signal Process. Mag., 16, 22-38 (1999).

[39] A. Aldroubi, M. Unser and M. Eden, "Cardinal spline filters: stability and convergence to the ideal sinc interpolator," Signal Process., 28, 127-138 (1992).

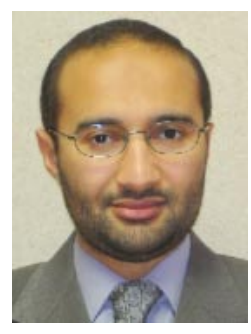

Ahmed Desoki received the B.S. and M.S. degrees in aerospace engineering from Cairo University, Egypt, in 2000 and 2004 respectively. He was with Aerospace Engineering Department, Cairo University as a Teaching Assistant from 2000 to 2004 and as a Lecturer Assistant from 2004 to present. Currently he is doctoral student in mechanical sciences and engineering at Tokyo Institute of Technology, Japan. His research interests are noise and vibration, digital signal processing, radar/sonar systems and CAE.

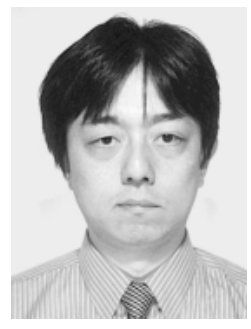

Jun-ichi Takada received the B.E., M.E., and D.E. degrees from Tokyo Institute of Technology, Tokyo, Japan, in 1987, 1989 and 1992, respectively. From 1992 to 1994 he was a Research Associate Professor with Chiba University, Chiba, Japan. From 1994 to 2006, he was an Associate Professor with Tokyo Institute of Technology. Since 2006, he has been a Professor with Tokyo Institute of Technology. His current interests are wireless propagation and channel modeling, array signal processing, UWB radio, cognitive radio, applied radio instrumentation and measurements, and ICT for international development. Dr. Takada is a member of IEEE, ACES, and the ECTI Association Thailand.

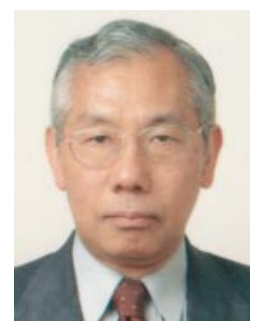

Ichiro Hagiwara received the B.S. and M.S. degrees in applied mathematics engineering from Kyoto University, Japan, in 1970, 1972 respectively, and the Ph.D. degree in mechanical engineering from the University of Tokyo, Japan in 1990. In 1972 he joined Nissan Motor Co., Ltd. He is a Professor with the Department of Mechanical Sciences and Engineering and Graduate School of Sciences and Engineering, Tokyo Institute of Technology since 1996 to present. He is also a Consulting Professor at Harbin Institute of Technology, P.R.China from 1998 to present, a Guest Professor and a Consulting Professor of the State Key Laboratory of Vibration, Shock and Noise of Shanghai Jiao Tong University, P.R.China from 1999 to present, a Honorary Professor of Tenjin University, China from 2005 to present and a Member of Science Council of Japan from 2006 to present. His main works are in $\mathrm{CAD} / \mathrm{CAM} / \mathrm{CAE} / \mathrm{CG}$, sound and vibration, robotics, origami engineering and collaboration engineering. Dr. Hagiwara is a member and Vice President of JSIAM, a fellow member of ASME, JSME and JSAE, Board member of JSST and a member of SICE, JSPE and JSCES. 